\title{
Stochasticity and Time Delays in Evolutionary Games
}

\author{
Jacek Miękisz • Sergiusz Wesołowski
}

Published online: 1 September 2011

(C) The Author(s) 2011. This article is published with open access at Springerlink.com

\begin{abstract}
We discuss combined effects of stochasticity and time delays in simple evolutionary games with a unique mixed evolutionarily stable strategy. We present three models of time-delay stochastic dynamics of finite well-mixed or random-matching populations. We show that in the first two models the evolutionarily stable strategy loses its stability and there appears a stable cycle around it with the time period and the amplitude proportional to the delay. In the third model, only one randomly chosen individual can update his strategy at a time. This slows down the dynamics and makes the evolutionarily stable strategy stable with respect to both time delay and stochastic perturbations.
\end{abstract}

Keywords Evolutionary game theory $\cdot$ Time delay $\cdot$ Stochastic dynamics $\cdot$ Stochastic stability $\cdot$ Stable cycles

\section{Introduction}

Many social and biological processes can be modeled as systems of interacting individuals within the framework of evolutionary game theory [3, 11, 15, 18, 23, 25, 26, 28]. One may then try to derive the macroscopic behavior of such populations from individual interactions. The evolution of very large (infinite) populations is usually modeled by differential or difference replicator equations which describe time changes of fractions of populations playing different strategies. For games with a unique mixed evolutionarily stable strategy, such as the Hawk-Dove game (also known as Chicken or Snowdrift game), the interior stationary point of replicator dynamics corresponding to such a strategy is globally asymptotically stable.

It is usually assumed (as in the replicator dynamics) that interactions between individuals take place instantaneously and their effects are immediate. In reality, all social and biological processes take a certain amount of time. It is natural therefore to introduce time delays into evolutionary game models. It is well known that time delays may cause oscillations in

\footnotetext{
J. Miękisz $(\bowtie) \cdot$ S. Wesołowski

Institute of Applied Mathematics and Mechanics, University of Warsaw, ul. Banacha 2, 02-097 Warsaw,

Poland

e-mail: miekisz@mimuw.edu.pl
} 
solutions of ordinary differential equations $[4,5,8-10,14]$. Effects of time delay in evolutionary games were discussed by Tao and Wang [24]. They considered a two-player game with two strategies and a unique mixed evolutionarily stable strategy. They proposed a certain form of a time-delay differential replicator equation and showed that the evolutionarily stable strategy is asymptotically stable for small time delays and that, for sufficiently large delays, it becomes unstable and there appear oscillations. However, as we pointed out in [1], its stability depends on particular causes of time delay. Models with time delays were divided into two families. In social-type models, where individuals react to the information concerning the state of the population at some earlier time, we should expect oscillations. On the other hand, in biological-type models, where some changes already take place in the population at an earlier time, oscillations might not be present for any time delay. In particular, we constructed two replicator-type dynamics. In the social-type model, we assumed that individuals at time $t$ imitate a strategy with a higher average payoff at time $t-\tau$ for some delay $\tau$. In the biological-type model, individuals born at time $t-\tau$ are able to take part in contests when they become mature at time $t$ or equivalently they are born $\tau$ units of time after their parents played and received payoffs. We showed that in the first dynamics, the evolutionarily stable strategy is asymptotically stable for small time delays and becomes unstable for big ones. In the second dynamics, however, it is asymptotically stable for any time delay.

Replicator equations describe the population behavior in the limit of an infinite number of individuals. However, real populations are finite. Stochastic effects connected with random matching of players, mistakes of players, and biological mutations can play a significant role in such systems. Therefore, to describe and analyze their time evolution, one should use stochastic modeling. Stochastic dynamics of well-mixed populations of a fixed number of players interacting in discrete moments of time were discussed in $[12,13,16,17,22$, $23,25,27]$. For symmetric games with two strategies, a state of the population is given by the number of individuals playing, say, the first strategy. The selection part of the dynamics ensures that if the average payoff of a given strategy is bigger than the average payoff of the other one, then the number of individuals playing the given strategy increases. In the model introduced by Kandori et al. [13], one assumes (as in the standard replicator dynamics) that individuals receive average payoffs weighted by fractions of different strategies present in the population. In the model introduced by Robson and Vega-Redondo [22], at any moment of time, individuals play only one or few games with randomly chosen opponents - there appears a noise connected with random matching of players. In both models, players may mutate with a small probability, hence the population may move against a selection pressure. To describe the long-run behavior of such stochastic dynamics, Foster and Young [6] introduced a concept of stochastic stability. A state of the system is stochastically stable if it has a positive probability in the stationary distribution of the corresponding Markov chain in the zero-noise limit, that is, the zero probability of mistakes or the zero-mutation level. It means that along almost any time trajectory the frequency of visiting this state converges to a positive value given by the stationary probability distribution.

For games with two strategies and two symmetric Nash equilibria, one of which is efficient and the other risk dominant, Kandori et al. [13] showed that in their model the riskdominant strategy is stochastically stable-we observe it in the long run with the frequency close to one if the mutation level is small enough. In the model of Robson and Vega-Redondo [22], the efficient strategy is stochastically stable. We have pointed out [16] that the long-run behavior of such evolutionary games depends on the relation between the noise and the size of the population. In the limit of zero noise, the efficient strategy is stochastically stable and in the limit of the infinite population, the risk-dominant one becomes stochastically stable. 
We see again that the long-run behavior of populations of individuals depends on details of the dynamics.

Here we will study how time delay affects the long-run behavior of the above models for two-strategy games with a unique evolutionarily stable strategy. In Sect. 2, we discuss a random walk with a time delay and show that an invariant cycle is stochastically stable. In Sect. 3, we discuss well-mixed and random-matching populations and show that, depending on details of the dynamics, either the unique evolutionarily stable strategy or a cycle around it is stochastically stable.

\section{Random Walk with a Time Delay}

To illustrate why time delay may cause oscillations, we discuss a simple discrete-time deterministic dynamical model with a finite number of states. Let a state of our system at time $t$ be described by $x(t) \in\{-m,-m+1, \ldots, 0, \ldots, m-1, m\}$, where $m$ is a natural number. A change of the state of the system at time $t+1$ depends on the state at some earlier time $t-\tau$, where $\tau$ is a discrete-time delay. We set

$$
x_{t+1}= \begin{cases}x_{t}+1 & \text { if } x_{t-\tau}<0 \\ x_{t}-1 & \text { if } x_{t-\tau}>0 \\ x_{t} & \text { if } x_{t-\tau}=0\end{cases}
$$

If $\tau=0$, then $x=0$ is the globally asymptotically stable stationary point of our dynamics. Consider now $0<\tau<m$. It is easy to see that in addition to the stationary point $x=0$, the cycle around 0 with the amplitude $\tau$ and the time period $4 \tau+2$ is a trajectory of our dynamics, that is, it is invariant under the rule (2.1). Moreover, when we start with any consistent initial condition $\left(x_{0}, x_{-1}, \ldots, x_{-\tau}\right)$, that is, $\left|x_{t}-x_{t-1}\right| \leq 1, t=0, \ldots,-\tau+1$ with not all $x$ 's equal to 0 , we end up in the cycle in a finite time. To see this let us first notice that at some moment $t$, the system has to be in some state $x,|x|>\tau / 2$. It means that $x_{t^{\prime}}, x_{t^{\prime}-1}, \ldots, x_{t^{\prime}-\tau}$ will all have the same sign at some future time $t^{\prime}$ and then the system will arrive immediately at the cycle.

Now let us perturb stochastically our dynamics. Namely, at every time step our system changes the state according to the rule (2.1) with the probability $1-\epsilon$ and with the probability $\epsilon$ it moves in the other direction; when $x_{t-\tau}=0$, then the system at time $t+1$ moves to the right or to the left with the probability $\epsilon / 2$; if $x_{t}= \pm m$, then $x_{t+1}=x_{t}$ with the probability $\epsilon$ and with the probability $1-\epsilon$ the system moves toward the origin. We obtained a random walk with reflecting boundaries and with a time delay, the simplest model involving both stochasticity and time delay. Figure 1a shows a trajectory of a deterministic walk starting with the initial condition $x_{t}=1$ for $t=0,-1, \ldots,-\tau$ and following the rule (2.1) with $m=20, \tau=10$; Fig. 1b shows a typical trajectory of the stochastic rule with $\epsilon=0.1$. One can see stochastic fluctuations around the deterministic globally stable cycle. Below we will analyze mathematically such behavior.

It was shown $[19,20]$ that in a time-delay random walk model without reflecting boundaries (that is, with infinite state space $Z$ ), the mean square displacement of the walker (that is, the variance) approaches a stationary value in an oscillatory way for large time delays and in a monotonic way for small ones. Moreover, this stationary value is a linear function of the delay.

Now we will redefine states of our stochastic dynamics to be $\tau+1$-tuples $\left(x_{t}, x_{t-1}, \ldots, x_{t-\tau}\right)$ at time $t$. Then our random walk with a time delay becomes a Markov 
a

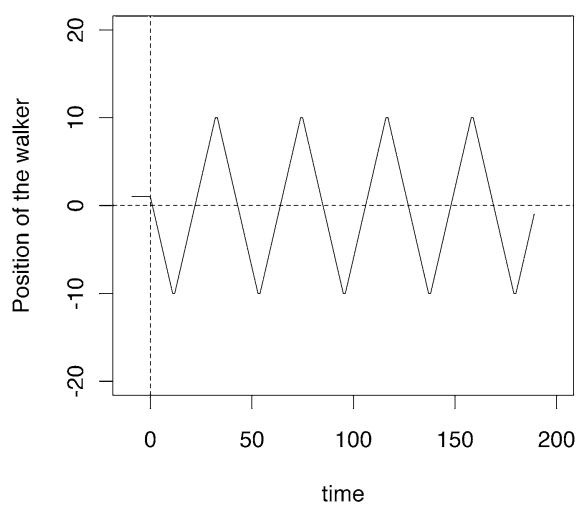

b

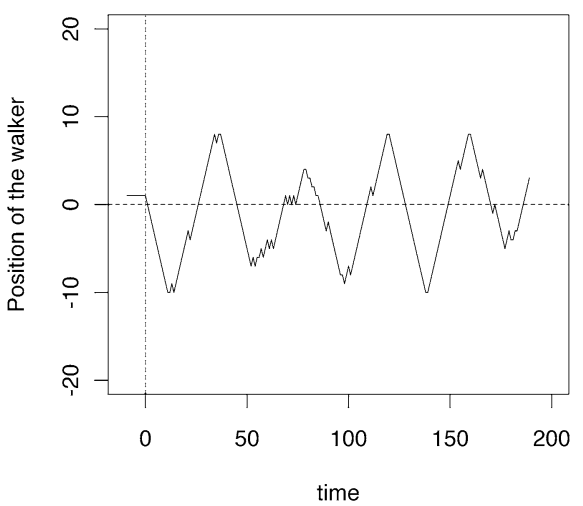

Fig. 1 Simulations of a random walk. (a) Deterministic trajectory, rule (2.1) with initial conditions $x_{t}=1, t=0, \ldots,-\tau, \tau=10$ and $m=20$. (b) A typical stochastic trajectory with $\epsilon=0.1$

chain with the unique stationary probability distribution denoted by $\mu_{\epsilon}$. Similar dynamical models with transition probabilities depending upon the finite history are known as highorder Markov chains [2, 21]. As was discussed in the beginning of this section, there are two absorbing sets of our dynamics with $\epsilon=0$ : the stationary state $(0, \ldots, 0)$ and the invariant cycle around 0 with the amplitude $\tau$ and the time period $4 \tau+2$. Now the question is which absorbing set survives small stochastic perturbations; that is, which one is in the support of the zero-mutation limit of the stationary probability distribution. The following concept of stochastic stability was introduced by Foster and Young [6].

Definition A subset $S$ of the state space of a Markov chain with the unique stationary probability distribution $\mu_{\epsilon}$ is stochastically stable if

$$
\lim _{\epsilon \rightarrow 0} \mu_{\epsilon}(S)=1
$$

It means that along almost any trajectory, for a small mutation level, the frequency of visiting $S$ converges to a value close to 1 .

We will now use the tree representation of the stationary probability distribution (see the Appendix) to prove that our cycle is stochastically stable. Let $c$ be a state which is a part of the cycle. Obviously all such $c^{\prime}$ s have the same probability in the stationary distribution.

Theorem $1 \lim _{\epsilon \rightarrow 0} \mu_{\epsilon}(c)=\frac{1}{4 \tau+2}$.

Proof It follows from the global asymptotic stability of the cycle that the system needs only one mutation to get out of $(0, \ldots, 0)$ and arrive at any state of the cycle. On the other hand, it needs $\tau$ mutations to get out of the cycle and arrive at $(0, \ldots, 0)$.

We have shown that the cycle which consists of $4 \tau+2$ consecutive states is stochastically stable in a simple random walk with a time delay. In the next section, we will discuss various time-delay stochastic evolutionary game dynamics and characterize their stochastically stable states. 


\section{Stochastic Evolutionary Models with Time Delays}

We will discuss here time-delay stochastic dynamics of fixed-size populations. Individuals are paired to play a Hawk-Dove game (also known as Chicken or Snowdrift game) with payoffs given by the following matrix:

$$
U=\left(\begin{array}{lll} 
& A & B \\
A & a & b \\
B & c & d
\end{array}\right)
$$

where $c>a$ and $b>d$ so there exists a unique mixed evolutionarily stable strategy, $x^{*}=$ $\frac{b-d}{c-a+b-d}$, which is the asymptotically stable stationary point of the replicator dynamics. It is interpreted as the equilibrium fraction of an infinite population playing $A$.

\section{Model 1}

We will consider a well-mixed population of $n$ players receiving average payoffs with respect to the composition of the population. Let $z_{t}$ be the number of individuals playing the strategy $A$ at a time $t$. The average payoffs of strategies $A$ and $B$ are given by

$$
\begin{aligned}
& \pi_{A}\left(z_{t}\right)=\frac{a\left(z_{t}-1\right)+b\left(n-z_{t}\right)}{n-1}, \\
& \pi_{B}\left(z_{t}\right)=\frac{c z_{t}+d\left(n-z_{t}-1\right)}{n-1},
\end{aligned}
$$

provided $0<z_{t}<n$.

It means that payoffs are average values weighted by fractions of $A$ and $B$ strategists. If $z=\frac{b-d}{c-a+b-d} n+\frac{d-a}{c-a+b-d}$, then average payoffs of $A$ and $B$ are equal. To avoid unnecessary technical complications due to a finite number of players, we assume that $z^{*}=n x^{*}$ is a natural number and $a=d$. It follows that if $z<z^{*}$, then $\pi_{A}(z)>\pi_{B}(z)$, if $z>z^{*}$, then $\pi_{A}(z)<\pi_{B}(z)$, and finally if $z=z^{*}$, then $\pi_{A}(z)=\pi_{B}(z)$.

The following dynamical rules are direct extensions of stochastic evolutionary dynamics introduced by Kandori et al. [13] to systems with time delays:

$$
z_{t+1}= \begin{cases}z_{t}+1 & \text { if } \pi_{A}\left(z_{t-\tau}\right)>\pi_{B}\left(z_{t-\tau}\right), \\ z_{t}-1 & \text { if } \pi_{A}\left(z_{t-\tau}\right)<\pi_{B}\left(z_{t-\tau}\right), \\ z_{t} & \text { if } \pi_{A}\left(z_{t-\tau}\right)=\pi_{B}\left(z_{t-\tau}\right),\end{cases}
$$

with the probability $1-\epsilon$ and with the probability $\epsilon$ the population moves in the other direction; if $\pi_{A}\left(z_{t-\tau}\right)=\pi_{B}\left(z_{t-\tau}\right)$, then the number of $A$-strategists decreases or increases by one at time $t+1$ with the probability $\epsilon / 2$; if $z_{t}=0$ or $n$, then $z_{t+1}=z_{t}$ with the probability $\epsilon$ and with the probability $1-\epsilon$ the system moves toward the interior stationary state. Our model is of the social type, players want to imitate a strategy with a higher average payoff at some earlier time. We do not need mutations to get out of a homogeneous state, in such states individuals would like to be different-the best reply to a given strategy is the other one. It is easy to see that we obtained a version of the random walk with a time delay discussed in Sect. 2. One can repeat the proof of Theorem 1 and show that our evolutionary dynamics with a time delay has a stochastically stable cycle around the evolutionarily stable strategy. 
a

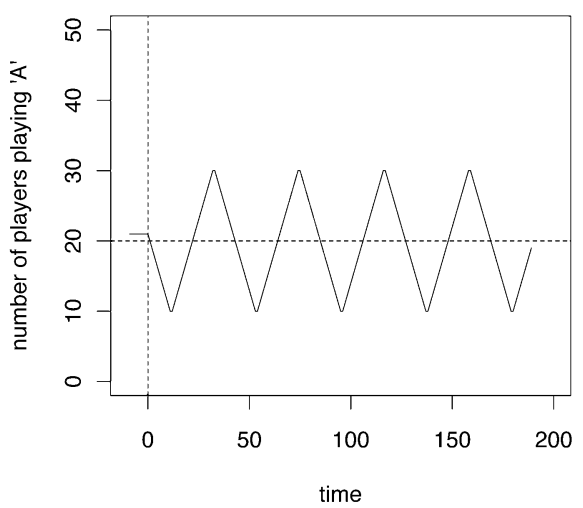

b

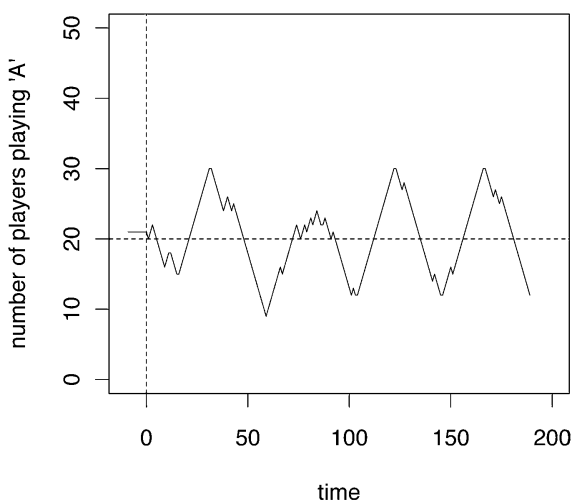

Fig. 2 Simulations of a random-matching population. (a) A typical mutation-free trajectory with initial conditions $x_{t}=1, t=0, \ldots,-\tau, \tau=10$ and $n=40$. (b) A typical stochastic trajectory with $\epsilon=0.1$

\section{Model 2}

Now we will consider a model introduced by Robson and Vega-Redondo [22]. Individuals are paired only once at every time step and play only one game before the imitation process takes place. Let $p_{t}$ denote the random variable which describes the number of cross-pairings, i.e. the number of pairs of matched individuals playing different strategies at the time $t$. Let us notice that $p_{t}$ depends on $z_{t}$. For a given realization of $p_{t}$ and $z_{t}$, average payoffs obtained by each strategy are as follows:

$$
\begin{aligned}
& \tilde{\pi}_{A}\left(z_{t}, p_{t}\right)=\frac{a\left(z_{t}-p_{t}\right)+b p_{t}}{z_{t}}, \\
& \tilde{\pi}_{B}\left(z_{t}, p_{t}\right)=\frac{c p_{t}+d\left(n-z_{t}-p_{t}\right)}{n-z_{t}},
\end{aligned}
$$

provided $0<z_{t}<n$.

The dynamical rules are the same as in (3.2), with $\pi_{A}$ and $\pi_{B}$ replaced by $\tilde{\pi}_{A}$ and $\tilde{\pi}_{B}$. Let us observe though that random matching introduces another sort of stochasticity. Therefore even without mutations $(\epsilon=0)$, the population may pass from the evolutionarily stable state to a cycle and back (unless a payoff matrix is symmetric as below and then we need one mutation to get out of the state $z^{*}=n / 2$ ); we do not have non-overlapping basins of attraction. Results of numerical simulations of our dynamics for a payoff matrix with $a=d=0$ and $b=c=1$ and $n=40$ are presented in Fig. 2. Like in Model 1 we see a stochastically perturbed cycle around $z^{*}$. It is not a surprise because for a big $n$, random-matching populations are well described by a well-mixed population model as was shown in Miękisz [16].

\section{Model 3}

Here we assume the set-up of well-mixed populations present in Model 1. The dynamical rules are, however, different. At any discrete moment of time, a randomly chosen player has a chance to revise his strategy. The number of $A$-players in the population may increase by one in $t+1$ if a $B$-player is chosen in $t$ which happens with the probability $\left(n-z_{t}\right) / n$. 

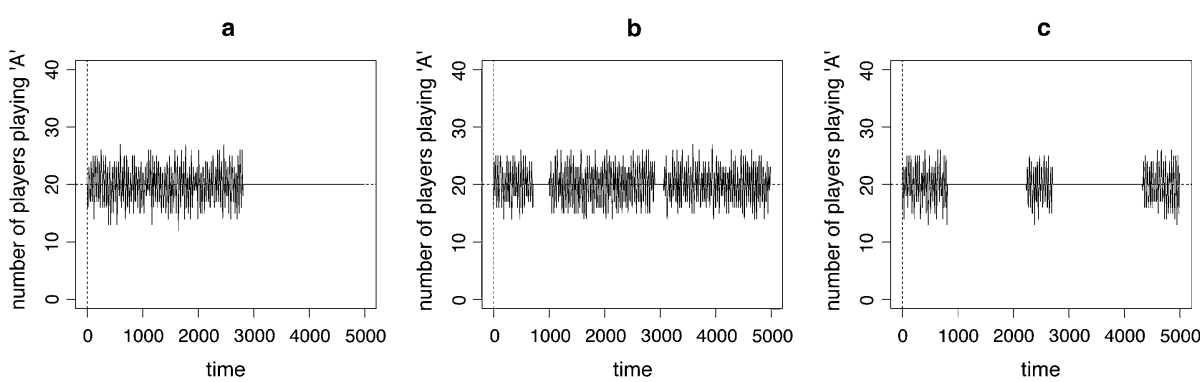

Fig. 3 Simulations of a random-player model. (a) A typical mutation-free trajectory with initial conditions $x_{t}=1, t=0, \ldots,-\tau, \tau=10$ and $n=40$. (b) A typical stochastic trajectory with $\epsilon=0.01$. (c) A typical stochastic trajectory with $\epsilon=0.001$

Analogously, the number of $B$-players in the population may increase by one in $t+1$ if an $A$-player is chosen in $t$ which happens with the probability $z_{t} / n$. The player, who has a revision opportunity, chooses in $t+1$ with the probability $1-\epsilon$ a strategy which had a higher average payoff at $t-\tau$ and the other one with the probability $\epsilon$. If both strategies provided the same average payoff at $t-\tau$, then we assume that the player will stay with the probability $1-\epsilon$ with his current strategy and with the probability $\epsilon$ he will adopt the other one. If at time $t-\tau$ only one strategy was present in the population, then at time $t$ a player chooses the other strategy with the probability $1-\epsilon$. Let us notice that even without mutations, the procedure of choosing a random player introduces stochasticity into the system. Now at any time $t$, a player can be chosen whose strategy had a higher payoff at time $t-\tau$. Then with the probability $1-\epsilon$, the population does not change. The time evolution of the populations is therefore slower than in previous models. This allows the evolutionarily stable strategy to be stable against time delays and small stochastic perturbations.

We will now use the tree representation of the stationary probability distribution (see the Appendix) to prove that $\left(z^{*}, \ldots, z^{*}\right)$ (that is, evolutionarily stable strategy) is stochastically stable. Let $\mu_{\epsilon}$ be the unique stationary probability distribution of the above described Markov chain.

Theorem $2 \lim _{\epsilon \rightarrow 0} \mu_{\epsilon}\left(z^{*}, \ldots, z^{*}\right)=1$

Proof It is easy to see that from every state we can arrive at $\left(z^{*}, \ldots, z^{*}\right)$ by the mutation-free dynamics, that is, the one with $\epsilon=0$. On the other hand, we need one mutation to get out of $\left(z^{*}, \ldots, z^{*}\right)$.

Figure 3 shows results of simulations. In the absence of mutations, the population settles at the evolutionarily stable state and stays there forever. For a small mutation level, we observe stochastic fluctuations around it. For smaller values of $\epsilon$, the population stays longer at the evolutionarily stable state; roughly 10 times longer in Fig. $3 c(\epsilon=0.001)$ than in Fig. $3 b(\epsilon=0.01)$. It follows from the rules of the dynamics that an amplitude of these fluctuations is proportional to the time delay.

\section{Discussion}

It is well known that time delays may cause oscillations in dynamical systems. We presented here three time-delay stochastic evolutionary game models with a unique mixed evolutionar- 
ily stable strategy. In the first two models (in well-mixed and random-matching populations), the evolutionarily stable strategy loses its stability and we obtain a stochastically stable cycle around it. In the third model, we allow only one randomly chosen individual to update his strategy at a time; hence, the time evolution is slower than in previous models. This allows the evolutionarily stable strategy to be stable against time delays and small stochastic perturbations. It is important to study combined effects of time delays and stochasticity in more complex evolutionary systems, and in particular the possibility of stable oscillations.

Acknowledgements Some of the results presented here were obtained in the Master thesis written by Jan Alboszta under the supervision of J.M. J.M. would like to thank the Polish Ministry of Science and Higher Education for a financial support under the grant N N201 362536.

Open Access This article is distributed under the terms of the Creative Commons Attribution Noncommercial License which permits any noncommercial use, distribution, and reproduction in any medium, provided the original author(s) and source are credited.

\section{Appendix: Stationary Probability Distributions of Ergodic Markov Chains}

The following tree representation of a unique stationary probability distribution of an ergodic Markov chain was proposed by Freidlin and Wentzell [7]. Their formula is especially useful in the case of random perturbations of deterministic dynamics on a finite state space. We assume that the system follows some deterministic rule with the probability $1-\epsilon$ and with the probability $\epsilon$, a mistake is made that moves the system in the other direction.

Let $(\Omega, P)$ be an ergodic Markov chain with a state space $\Omega$ and transition probabilities given by $P_{\epsilon}: \Omega \times \Omega \rightarrow[0,1]$. It has the unique stationary probability distribution $\mu_{\epsilon}$. For $x \in \Omega$, an $x$-tree is a directed graph on $\Omega$ (connecting all vertices) such that from every $y \neq x$ there is a unique path to $x$ and there are no outcoming edges out of $x$. Denote by $T(x)$ the set of all $x$-trees and let

$$
q_{\epsilon}(x)=\sum_{d \in T(x)} \prod_{\left(y, y^{\prime}\right) \in d} P_{\epsilon}\left(y, y^{\prime}\right)
$$

where $P_{\epsilon}\left(y, y^{\prime}\right)$ is the element of the transition matrix (that is, a conditional probability that the system will be at the state $y^{\prime}$ at time $t$ provided it was at state $y$ at time $t-1$ ) and the above product is with respect to all edges of the $x$-tree $d$. In all models of our paper, $P_{\epsilon}\left(y, y^{\prime}\right)$ is equal either to $1-\epsilon, \epsilon$, or 0 . Now one can show that

$$
\mu_{\epsilon}(x)=\frac{q_{\epsilon}(x)}{\sum_{y \in \Omega} q_{\epsilon}(y)}
$$

for all $x \in \Omega$.

A state is an absorbing one if it attracts nearby states in the mutation-free dynamics $(\epsilon=0)$. We assume that after a finite number of steps of the mutation-free dynamics, the system arrives at one of the absorbing states and stays there forever. It follows from (5.2) that the stationary probability distribution can be written as the ratio of two polynomials in $\epsilon$. Hence any non-absorbing state has zero probability in the stationary distribution in the zero-mutation limit. Moreover, in order to study the zero-mutation limit of the stationary distribution, it is enough to consider paths between absorbing states. Let $m_{x y}$ be a minimal number of mutations (mistakes) needed to make a transition from the state $x$ to $y$ and $m_{y x}$ the minimal number of mutations to make a transition from $y$ to $x$. Then $q_{\epsilon}(x)$ is of the order $\epsilon^{m_{y x}}$ and $q_{\epsilon}(y)$ is of the order $\epsilon^{m_{x y}}$. If for example, $m_{y x}<m_{x y}$, then it follows that $\lim _{\epsilon \rightarrow 0} \mu_{\epsilon}(x)=1$, hence $x$ is stochastically stable. 


\section{References}

1. Alboszta J, Miękisz J (2004) Stability of evolutionarily stable strategies in discrete replicator dynamics. J Theor Biol 231:175-179

2. Ching WK, Ng MK, Fung ES (2008) Higher-order multivariate Markov chains and their applications. Linear Algebra Appl 428:492-507

3. Cressman R (2003) Evolutionary dynamics and extensive form games. MIT Press, Cambridge

4. Erneux T (2009) Applied delay differential equations. Springer, New York

5. Foryś U (2004) Biological delay systems and the Mikhailov criterion of stability. J Biol Syst 12:1-16

6. Foster D, Young PH (1990) Stochastic evolutionary game dynamics. Theor Popul Biol 38:219-232

7. Freidlin MI, Wentzell AD (1984) Random perturbations of dynamical systems. Springer, New York

8. Gopalsamy K (1992) Stability and oscillations in delay differential equations of population. Springer, Berlin

9. Györi I, Ladas G (1991) Oscillation theory of delay differential equations with applications. Clarendon, Oxford

10. Hale J (1997) Theory of functional differential equations. Springer, New York

11. Hofbauer J, Sigmund K (1998) Evolutionary games and population dynamics. Cambridge University Press, Cambridge

12. Kamiński D, Miękisz J, Zaborowski M (2005) Stochastic stability in three-player games. Bull Math Biol 67:1195-1205

13. Kandori M, Mailath GJ, Rob R (1993) Learning, mutation, and long-run equilibria in games. Econometrica 61:29-56

14. Kuang Y (1993) Delay differential equations with applications in population dynamics. Academic Press, San Diego

15. Maynard Smith J (1982) Evolution and the theory of games. Cambridge University Press, Cambridge

16. Miękisz J (2005) Equilibrium selection in evolutionary games with random matching of players. J Theor Biol 232:47-53

17. Miękisz J (2008) Evolutionary game theory and population dynamics. In: Capasso V, Lachowicz M (eds) Multiscale problems in the life sciences, from microscopic to macroscopic. Lecture notes in mathematics, vol 1940, pp 269-316

18. Nowak MA (2006) Evolutionary dynamics: exploring the equations of life. Harvard University Press, Cambridge

19. Ohira T, Milton JG (1995) Delayed random walks. Phys Rev E 52:3277-3280

20. Ohira T (1997) Oscillatory correlation of delayed random walks. Phys Rev E 55:R1255-R1258

21. Raftery AE (1985) A model for high-order Markov chains. J R Stat Soc B 47:528-539

22. Robson A, Vega-Redondo F (1996) Efficient equilibrium selection in evolutionary games with random matching. J Econ Theory 70:65-92

23. Sandholm WH (2010) Population games and evolutionary dynamics. MIT Press, Cambridge

24. Tao Y, Wang Z (1997) Effect of time delay and evolutionarily stable strategy. J Theor Biol 187:111-116

25. Vega-Redondo F (1996) Evolution, games, and economic behaviour. Oxford University Press, Oxford

26. Weibull J (1995) Evolutionary game theory. MIT Press, Cambridge

27. Young PH (1993) The evolution of conventions. Econometrica 61:57-84

28. Young HP (1998) Individual strategy and social structure: an evolutionary theory of institutions. Princeton University Press, Princeton 